\title{
ARTICLE \\ Single cell and tissue-transcriptomic analysis of murine bladders reveals age- and TNF $\alpha$-dependent but microbiota-independent tertiary lymphoid tissue formation
}

\author{
Marianne M. Ligon ${ }^{1}$, Caihong Wang ${ }^{1}$, Erica N. DeJong ${ }^{2}$, Christian Schulz ${ }^{2}$, Dawn M. E. Bowdish ${ }^{2}$ and Indira U. Mysorekar (iD ${ }^{1,3}$
}

\begin{abstract}
Aging has multifaceted effects on the immune system, but how aging affects tissue-specific immunity is not well-defined. Bladder diseases characterized by chronic inflammation are highly prevalent in older women, but mechanisms by which aging promotes these pathologies remain unknown. Tissue transcriptomics of unperturbed, young and aged bladders identified a highly altered immune landscape as a fundamental feature of the aging female bladder. Detailed mapping of immune cells using single cell RNAsequencing revealed novel subsets of macrophages and dendritic cells and unique changes to the immune repertoire in the aged bladder. $\mathrm{B}$ and $\mathrm{T}$ cells are highly enriched in aged bladders and spontaneously form organized bladder tertiary lymphoid tissues (bTLTs). Naïve, activated, and germinal center B cells and $\lg A^{+}$plasma cells are found within bTLT and associated with increased urinary lgA concentrations. bTLTs form with increasing age and their formation is dependent on TNFa. Microbiota are not required to form bTLT, as aged germfree mice also harbor them. Thus, bTLTs require age-dependent TNFa but are independent of the microbiota. Our results indicate that chronic, age-associated inflammation underlies a fundamental alteration to the bladder and establishes a resource for further investigation of the bladder immune system in homeostasis, aging, and disease.
\end{abstract}

Mucosal Immunology (2020) 13:908-918; https://doi.org/10.1038/s41385-020-0290-x

\section{INTRODUCTION}

Immune dysfunction during aging is characterized by chronic, low-grade inflammation, termed inflamm-aging. ${ }^{1}$ Aging is the strongest risk factor for many chronic diseases, including cardiovascular disease, neurodegeneration, osteoarthritis, and cancers. ${ }^{2}$ While these diseases are all linked by chronic inflammation, immune responses vary by tissue, resulting in tissue-specific immune dysfunction. ${ }^{3}$

The bladder is a storage organ with a mucosal barrier that provides protection from both urinary wastes and pathogens. ${ }^{4,5}$ Bladder diseases are highly prevalent among the elderly, and women are predominately affected by these diseases. ${ }^{6-9}$ Older women $(50+)$ are highly susceptible to bladder disorders including overactive bladder/urge incontinence (OAB), interstitial cystitis/bladder pain syndrome (IC/BPS), and recurrent urinary tract infections (rUTIs). These disorders all have a chronic inflammatory component as well as overlapping symptoms, known as lower urinary tract symptoms (LUTS). ${ }^{10-12}$ How and why these bladder diseases become more prevalent with aging is not currently understood. ${ }^{9}$ Given the common association of bladder diseases with both aging and chronic inflammation, local immune dysfunction at the bladder mucosa may underlie mechanisms driving age-related bladder diseases.

During homeostasis, bladder immune cells consist of $\sim 80 \%$ antigen-presenting macrophages and dendritic cells (DCs), $10 \%$ $\mathrm{T}$ cells, and small numbers of NK cells, mast cells, eosinophils, and patrolling monocytes. ${ }^{13,14}$ Whether aging affects immunity in the bladder mucosa is not known; however, since chronic bladder inflammation is highly prevalent in older women, age-associated disruption of immune homeostasis in the bladder may mediate inflammatory pathology and LUTS.

The bladder lacks dedicated mucosal secondary lymphoid organs (SLOs) that form during development like the Peyer's patches of the small intestine, resulting in a uniquely quiescent bladder mucosal immune system. Non-lymphoid organs may sometimes form ectopic, SLO-like structures in response to chronic inflammation and antigen exposure. ${ }^{15-17}$ Lymphoid aggregates have been reported in cases of chronic bacteriuria, muscleinvasive bladder cancer, and IC/BPS. ${ }^{18-20}$ However, lymphoid aggregates in the bladder remain largely uncharacterized and are not reported to spontaneously form in the mouse bladder. Furthermore, factors influencing their formation, composition, or persistence, have not been identified.

In this report, we sought to identify underlying effects of aging on bladder tissue and the bladder immune cell repertoire using global tissue transcriptomics and targeted, single-cell transcriptomics from young and aged mouse bladders. We find that aging fundamentally alters the bladder immune landscape on the cellular and tissue-transcriptomic levels. We demonstrate that in unperturbed aged bladders, expanded numbers of B and T cells organize into structures we term bladder tertiary lymphoid tissue (bTLT). bTLT in aged mice serve as centers for B cell recruitment, activation, and differentiation into plasma cells. Age-dependent bTLT form in aged germ-free mice, but aged $\mathrm{TNFa}^{-/-}$mice have

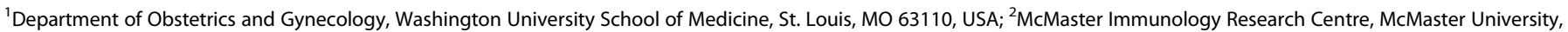
Hamilton, ON, Canada and ${ }^{3}$ Department of Pathology and Immunology, Washington University School of Medicine, St. Louis, MO 63110, USA

Correspondence: Indira U. Mysorekar (imysorekar@wustl.edu) 
fewer and smaller bTLT, indicating that bTLT require agedependent TNFa but are independent of the microbiota. Together these data reveal a profound change in the bladder mucosal immune system that is fundamental to aging in this tissue.

\section{RESULTS}

Tissue and single cell transcriptomics reveal substantial effects of aging on the bladder immune system

Older age is associated with many bladder diseases, yet little is known about the fundamental changes to the bladder that occur during aging. Since women have a higher prevalence of bladder disorders and have distinct lower urinary tract anatomy, physiology, and age-related changes from men, ${ }^{21}$ we chose to study how aging affects the bladder using aged female mice as a model. We first sought to characterize age-related global transcriptomic changes to the bladder by performing RNAsequencing (RNA-seq) on whole bladder tissue from young ( 3 month old) and aged (18 month old) female mice. Bladders from aged mice had at least a two-fold increase in expression of 416 genes and decrease in expression of 60 genes compared with those from young mice (Fig. 1a, Benjamini-Hochberg adjusted false discovery rate (FDR) $\leq 0.05$, Supplementary Table 1$)$. We then performed pathway analysis of all expressed genes using the Kyoto Encyclopedia of Genes and Genomes pathway database and identified 13 upregulated pathways and 1 downregulated pathway in the bladder transcriptome of aged mice compared with those of young mice (Fig. 1b). Highly upregulated pathways included antigen presentation, B- and T-cell activation, cytokine-receptor interactions, and intestinal $\lg A$ production (Fig. 1b, c). Enrichment of these pathways suggested that immune cells may be recruited to and activated within the aging bladder. Using independent samples, we validated increased expression of the most highly upregulated chemokine, $C_{x C l 13}$ (Fig. 1d), a homeostatic lymphoid chemokine that attracts naive lymphocytes to organized lymphoid tissues. We examined expression of the three other homeostatic lymphoid chemokines, finding that $\mathrm{Cl} / 19$, but not $\mathrm{CxCl} 12$ or $\mathrm{CCl} 21$, was also upregulated in aged bladders (Figs. 1d, Supplementary Fig. 1). In mice, bladder tissue typically harbors a small number of immune cells compared with other mucosal barriers, and many of these cells have unknown functions in bladder homeostasis or disease. ${ }^{4,13,22}$ Using flow cytometry, we found that aged bladders contained nearly three times as many $\mathrm{CD}_{4} 5^{+}$cells as young bladders (Fig. 1e), further indicating that the tissue transcriptomic changes we observed were likely due to high numbers of immune cells in aged bladders compared with young bladders. Notably, Tnf was also among the most highly upregulated cytokines in the aged bladder (Fig. 1c). TNFa plays a role in driving inflamm-aging and is a predictor of frailty and mortality in humans. ${ }^{23,24}$ Along with chemokine-mediated recruitment, locally increased TNFa in the bladder could be driving an age-associated inflammatory environment and activating immune cells in the aged bladder. Since the bladder mucosa is normally immunologically quiescent, these data suggested that an altered immune landscape was fundamental to the aging bladder.

To take an unbiased approach to identifying the immune cell types that were driving the immune cell accumulation and tissue transcriptomic changes in aged bladders, we performed dropletbased single-cell RNA-seq (scRNA-seq) on enriched $\mathrm{CD} 45^{+}$cells isolated from young and aged bladders. After removing urothelial and stromal cells from analysis, we identified 21 distinct immune cell clusters (Figs. 2a, Supplementary Figs. 2, 3). Using canonical cell type markers and queries against the ImmGen database of immune cell transcriptomes, ${ }^{25,26}$ we assigned identities to all of the immune cell clusters (Table 1, Supplementary Tables 2, 3). We identified immune cells that have been previously reported in bladder tissue, including macrophages, monocytes, DCs, natural killer cells, $\gamma \delta T$ cells, and both $\mathrm{CD}^{+}$and $\mathrm{CD}^{+}$aßT cells. ${ }^{13,14,27,28}$
In both young and aged bladders, we identified two distinct macrophage clusters distinguished by high expression of Retnla in the smaller cluster (Supplementary Fig. 4a). Retnla (also known as Relma or Fizz1) is a marker of alternatively activated macrophages; thus, this macrophage cluster may have a more pronounced tissue-reparative phenotype compared with the larger macrophage cluster. Interestingly, a small, third cluster of macrophages were exclusively found in the aged bladder (Figs. 2b, Supplementary Fig. 4b) and were distinguished by high expression of $\mathrm{CxCl} 13$ (Fig. 2c), one of the most highly upregulated genes identified by tissue RNA-seq. While there were not surface markers to distinguish these cells, we were able to detect highly increased expression of $\mathrm{CxCl} 13$ in sorted $\mathrm{F} 4 / 80^{+} \mathrm{CD} 4^{+} \mathrm{Ly} 6 \mathrm{C}^{-}$macrophages from aged mice compared with those from young mice (Fig. $2 \mathrm{~d}$ ). Thus, macrophages are the most likely source of the high Cxcl13 expression identified in the tissue transcriptome of the aged bladder and likely contribute to immune cell recruitment during aging. We also identified a distinct cluster of type 1 classical DCs (CDC1), which most likely correlate to previously reported CD103 ${ }^{+}$ DCs, and three clusters of type 2 CDC (CDC2), likely corresponding with previously reported CD11 b ${ }^{+}$DCs. ${ }^{13,29}$ However, we were unable to detect mast cells, which are known to play important roles in bladder inflammation and infection, or eosinophils, which have also been previously reported within mouse bladder tissue. ${ }^{13,29,30}$ These cells may not have been isolated by our methods or the populations may too small to resolve by clustering since they are known to be minor immune cell populations within the bladder. Our transcriptional analysis of the bladder immune repertoire at single-cell resolution is generally consistent with the literature while also identifying novel subsets of cells that may play distinct roles in bladder homeostasis, aging, and disease.

The most striking difference in the immune cell repertoire between young and aged bladders was a dramatic increase in B and $T$ cells within aged bladders (Fig. 2b, Supplementary Fig. 4b). $B$ cells are generally absent from young bladders, and $T$ cells comprise only a small number of cells in young bladders. ${ }^{13}$ The largest cluster of B cells were naive B cells marked by strong lghd expression (Supplementary Figs. 3, 4c). Two smaller B cell clusters expressed markers of activated B cells, such as Ctla4 and Mzb1 (Supplementary Fig. 4c). In one B cell cluster, 95\% of cells expressed a specific immunoglobulin germ-line variable gene (Supplementary Figs. 3, 4c, Supplementary Table 2), suggesting that some of these B cells may have been clonally expanded. Both $B$ cell clusters also expressed Fcrl5 (Supplementary Fig. 4c), a marker of dysfunctional B cells, termed age-associated B cells $(A B C s)$, which have been implicated in inflamm-aging and autoimmune disorders. The exact function of $A B C s$ is debated due to their similarity to a subset of atypical memory $B$ cells. ${ }^{31,32}$ scRNA-seq also demonstrated an increase in plasma cells in aged bladders. Plasma cells expressed high levels of Jchain (Supplementary Fig. 4c), which forms part of the multimeric, secretory forms of IgM and IgA. Thus, a spectrum of $B$ cell states, including naive, activated, and differentiated $B$ cells, were present in the aged bladder and could potentially be participating in active, local immune responses.

\section{Lymphocytes in aged mouse bladders organize into tertiary lymphoid tissues}

Since aging resulted in a substantial increase of lymphoid cells in the bladder according to scRNA-seq analysis, and little is known about these cell types in the bladder, we further investigated these cells in aged bladders. Flow cytometry confirmed that aged bladders indeed harbored a significant increase in total $T$ cells, $\mathrm{CD}^{+}$and $\mathrm{CD}^{+}{ }^{+} \mathrm{T}$ cell subsets, and B cells compared with young bladders (Fig. 3a). To investigate how the influx of lymphocytes affected the bladder tissue architecture, we examined bladders histologically. Surprisingly, we found that the majority of aged bladders contained large aggregates of lymphocytes in the lamina 


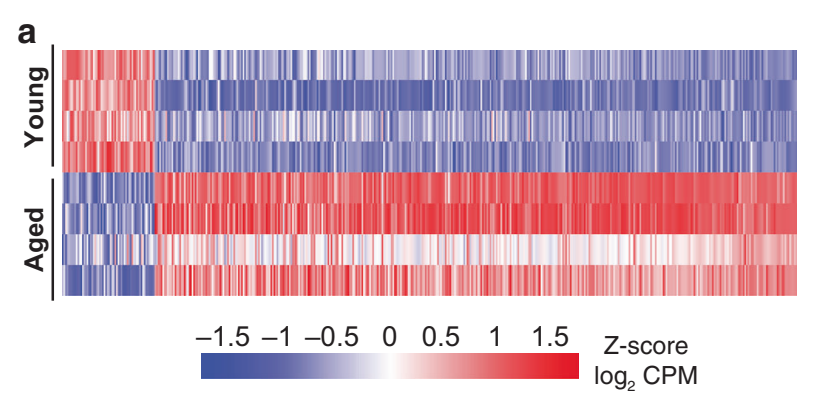

C
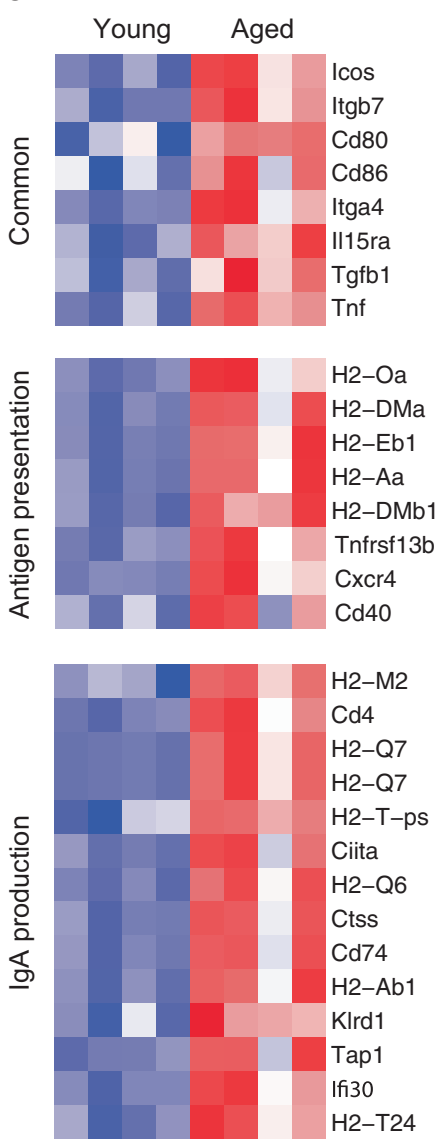

b

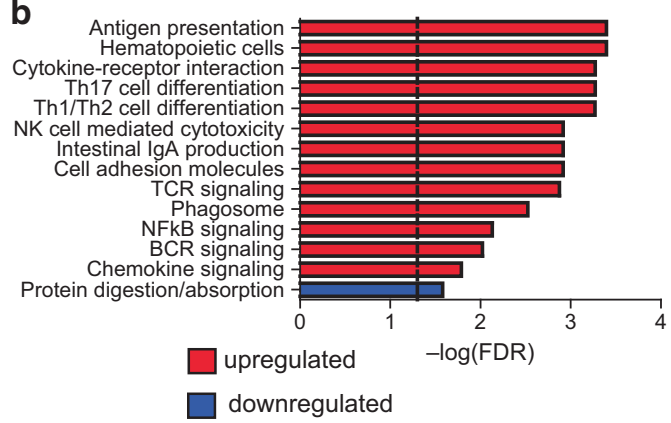

d

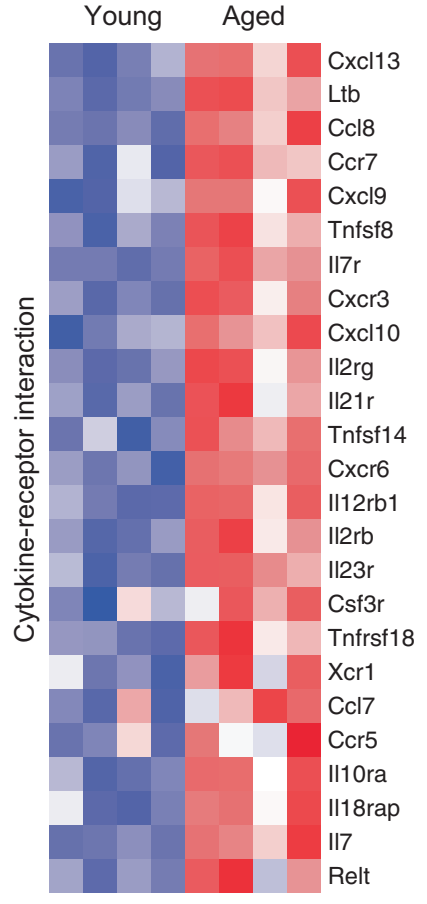

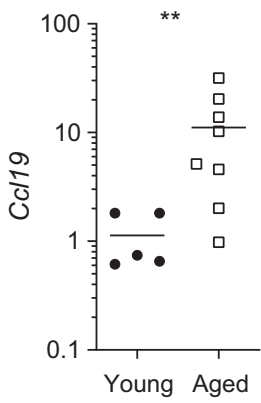

e

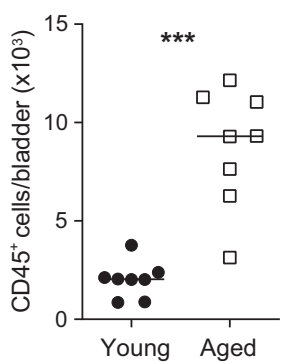

Fig. 1 Tissue transcriptomic map of young and aged bladders. a Heatmap of the global tissue transcriptome of young and aged bladders with fold change $>2$ and Benjamini-Hochberg FDR-adjusted $p<0.05 . n=4$ bladders/group. $\mathbf{b}$ Significantly enriched KEGG pathways based on fold change of all genes. c Heatmaps of significantly different genes from selected significant KEGG pathways. $\mathbf{d}$ Gene expression validation by qRT-PCR in whole bladders ( $n=5-11 /$ group). e Number of CD45 cells/bladders from young and aged mice $(n=8 /$ group) determined by flow cytometry. Line at median. ${ }^{* * *} p<0.001,{ }^{* *} p<0.01$, Mann-Whitney U-test.

propria that were completely absent in young bladders (Fig. 3b). Since aging is a continuous and highly variable process, we examined bladders from mice aged 3 to 18 months (m) (Fig. 3c). We found that lymphoid aggregates first appeared in some bladders at $9 \mathrm{~m}$, and all bladders contained these structures at 15 $\mathrm{m}$. These observations indicated that lymphoid aggregates began to form in "middle" age and became a common feature of mouse bladders by $18 \mathrm{~m}$ (approximately equivalent to 60 years old in humans). These structures led us to hypothesize that the $B$ and T cells accumulating in the bladder during aging were accumulating within these aggregates. Indeed, bladder lymphoid aggregates in aged mice were primarily composed of $\mathrm{CD}^{+} \mathrm{T}$ and $\mathrm{B}_{2} 20^{+} \mathrm{B}$ cells, while these cells were not found elsewhere in the lamina propria (Fig. 3d). In larger aggregates, B and T cells tended to segregate into respective zones, reminiscent of the highly organized structure of lymphoid tissues, such as lymph nodes. ${ }^{15,16}$ Lymphoid tissues contain specialized structures that facilitate their form and function in generating $B$ cell responses. In the aged bladder, we identified $\mathrm{CD} 31^{+}$(platelet endothelial cell adhesion molecule) vessels that also expressed peripheral node addressin (PNAd), a specific marker of specialized, high endothelial venules (HEVs) (Fig. 3e). HEVs are otherwise only found in lymphoid tissues and facilitate the circulation of naive lymphocytes into the structure. We also found CD35 ${ }^{\text {hi }}$ (complement receptor 1) follicular dendritic cell (FDC) networks within larger aggregates (Fig. 3f). FDCs are stromal cells that structurally support the organization of lymphoid tissues. These findings demonstrate that aged bladders, but not young bladders, contain lymphoid aggregates with features of organized lymphoid tissue; thus, hereafter we term these structures bladder tertiary lymphoid tissues (bTLTs). TLTs 
a

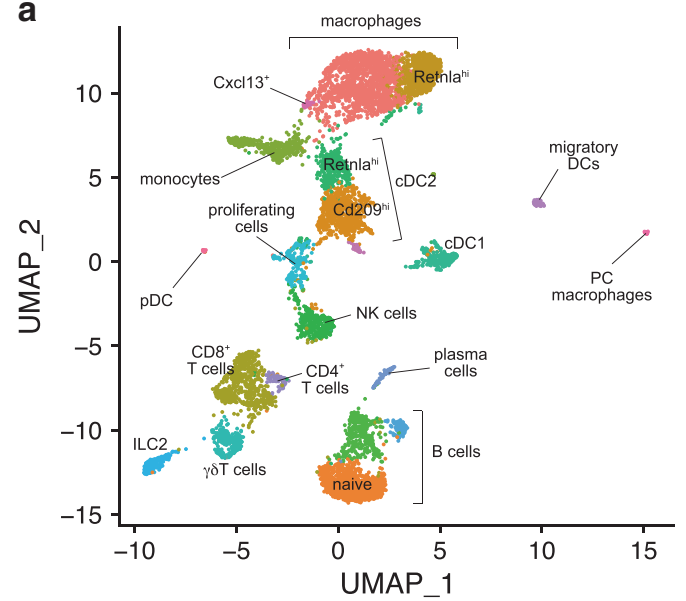

0 Bladder macrophages

1 Naive $B$ cells

2 CD209hi cDC2

- 3 Retna ${ }^{\text {hi }}$ macrophage

$4 \mathrm{CD}^{+} \mathrm{T}$ cells

5 Monocytes

6 B cells

- 7 NK cells

8 Retnlahi $c D C 2$

$9 \mathrm{cDC} 1$

- $10 \gamma \delta \mathrm{T}$ cells

11 Proliferating cell

12 ILC2

13 B cells

14 Plasma cells

$15 \mathrm{CD}^{+} \mathrm{T}$ cells

16 Migratory DCs

- $17 \mathrm{cDC} 2$

$18 \mathrm{Cxcl} 13^{+}$macrophages

19 Peritoneal cavity macrophages

20 pDC b

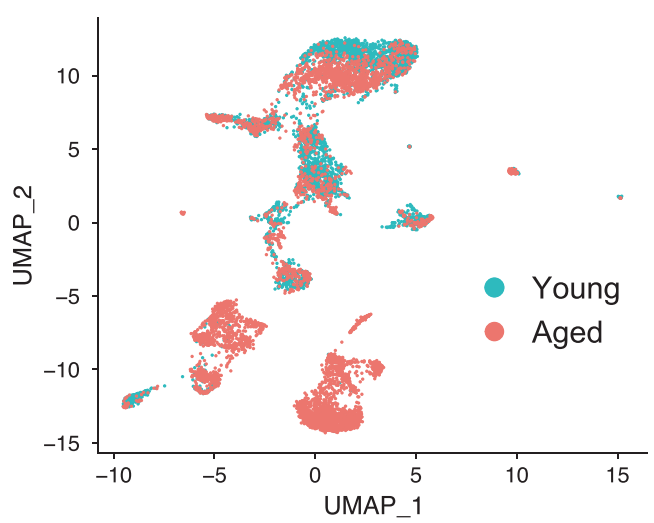

C

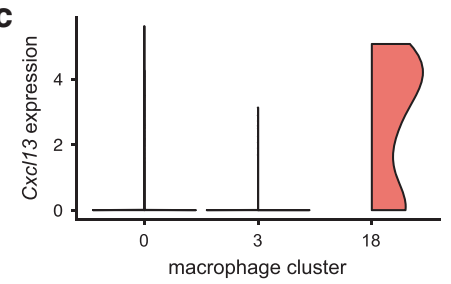

d

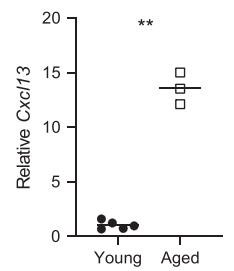

Fig. 2 Single cell transcriptomic map of immune cells from young and aged bladders. a Clustering analysis and cell type identification of CD45+ cells from young $(n=3209)$ and aged bladders $(n=4682)$. Cluster numbers are ordered by most abundant cell type from the merged dataset. b Map of cells originating from young or aged bladders. c Expression of $\mathrm{Cxcl13}$ in scRNA-seq macrophage clusters (numbered as in a and colored as in b). d RT-qPCR of sorted F4/80 ${ }^{+} \mathrm{CD} 64^{+}$Ly $6 \mathrm{C}^{-}$macrophages from young and aged mice $(n=3-5 / g r o u p) .{ }^{* *} p<0.01$, Unpaired $t$-test with Welch's correction. Lines at mean.

typically elaborate functions that otherwise only occur within the SLOs, but TLTs are localized to the site of inflammation and immune response. ${ }^{15,16}$ Lymphoid aggregates have been reported in a few models of chronic bacteriuria and bladder cancer but remain poorly characterized and have thus far not been reported in unperturbed bladders. ${ }^{18,19,33}$ Since bTLTs are specifically found in aged bladders, they could facilitate inflamm-aging within the bladder tissue and significantly alter immune responses to a variety of stimuli. To begin to understand these structures, we sought to further characterize them and determine their functional capacity during aging.

bTLTs contain germinal centers and promote class-switched $\lg \mathrm{A}$ responses

Since our scRNA-seq data indicated the presence of a range of $B$ cell states, we hypothesized that there were active $B$ cell responses occurring within bTLTs. Like their SLO counterparts, TLTs can support germinal center (GC) reactions where B cells are activated by antigen recognition, may undergo further selection, affinity maturation, and class-switch recombination, and differentiate into plasma cells that secrete large quantities of antibodies. ${ }^{34,35}$ We found that a large number of B cells in bTLTs were $\operatorname{lgD}^{+}$naive $B$ cells (Fig. $4 a$ ), consistent with our scRNA-seq data. Locally active GCs were identified within bTLTs by the highlyspecific GC marker GL-7 (Fig. 4b). We confirmed the presence of increased numbers of $\mathrm{CD}_{138^{+}}$(Syndecan- ${ }^{+}$) plasma cells by flow cytometry (Fig. 4c) and localized them to bTLT (Fig. 4d), indicating that differentiated $B$ cells were also part of these structures. While the majority of plasma cells identified by scRNA-seq expressed Ighm (Supplementary Fig. 2c), the presence of GCs within bTLTs suggested that class switch recombination (CSR) may occur locally. Furthermore, we demonstrated that larger bTLTs contained GCs where CSR typically takes place. Tissue RNA-seq also indicated that multiple class-switched isotypes were enriched within the aged bladder (Fig. 4e). The most highly enriched, class-switched isotype was $\lg \mathrm{A}$, which is the major secretory isotype. This finding agreed with the enriched intestinal IgA production pathway we previously identified in aged bladders (Fig. 1b), which includes processes that typically occur at Peyer's patches in the intestinal mucosa. $\lg \mathrm{A}$ is found in urine and polymeric Ig receptor (plgR), which transports secretory $\lg A$ across epithelial barriers, is expressed in the urothelium. ${ }^{36,37}$ However, little is known about the local production, transport, and regulation of IgA at the bladder mucosa. To determine if increased $\lg A$ was locally produced in the aging bladder, we first measured IgA concentration in the urine. Urine from aged mice contained approximately tenfold higher concentrations of IgA compared with urine from young mice (Fig. 4f). In the kidney, IgA transport by plgR is decreased during aging, which may contribute to IgA deposition at the glomerulus. ${ }^{38}$ Pigr was not identified as significantly up- or down-regulated in our tissue RNAseq data, and independent quantification of Pigr expression in bladders from young and aged mice did not show any significant 
Single cell and tissue-transcriptomic analysis of murine bladders reveals...

MM Ligon et al.

912

Table 1. Markers used to identities cell cluster identities from scRNA-seq.

\begin{tabular}{|c|c|c|}
\hline Cluster number/name & Cell identity markers & Top CIPR ${ }^{25,26}$ match \\
\hline 0 Macrophages & Adgre1 & Interstitial macrophage (lung) \\
\hline 1 B cells (naïve) & Cd79a, Ms4a1, lghd & Spleen follicular B cell \\
\hline 3 Macrophages (Retnla $\left.{ }^{\text {hi }}\right)$ & Adgre & Interstitial macrophage (lung) \\
\hline 4 CD8 $+\mathrm{T}$ cells & $C d 3 d, C d 3 g, C d 3 e, C D 8 b 1$, Gzmk & Naïve CD8 $+\mathrm{T}$ cell $96 \mathrm{~h}$ after in vitro stimulation \\
\hline 7 NK cells & Gzma, Gzmb & Splenic NK cell Ly49H+ subset \\
\hline 8 cDC2 (Retnla $\left.{ }^{\text {hi }}\right)$ & Sirpa, Mgl2 & Adipose tissue CD11b+ DC \\
\hline $9 \mathrm{cDC} 1$ & Flt3, Xcr1, Clec9a & Adipose tissue CD11b- DC \\
\hline $10 \gamma \delta \mathrm{T}$ cells & Tcrd, Tcrg, Tcre & Thymus B6 E15 TCRgammadelta \\
\hline 11 Proliferating cells & Mki67, Top2a, Ube2c & Pre-T cell double positive blasts ${ }^{a}$ \\
\hline 15 CD4+ T cells & $C d 3 d, C d 3 g, C d 3 e, C d 4$ & CD4+ $T$ cell 8 days after $L C M V$ infection \\
\hline 16 Migratory DCs & Flt3, Fscn1, Cacnb3, Nudt17, Socs2 ${ }^{64}$ & Skin draining lymph node DC \\
\hline $17 \mathrm{cDC} 2$ & Flt3, Sirpa, Mgl2 & Thymus double negative DC \\
\hline 18 Macrophages $\left(\mathrm{C} x \mathrm{C} / 13^{+}\right)$ & Adgre1 & F4/80hi liver macrophage \\
\hline 19 Macrophages (peritoneal) & Adgre1 & Peritoneal macrophage steady state \\
\hline $20 \mathrm{pDC}$ & Flt3, Pacsin1, Siglech, Irf8, Cox6a2, Run $\times 2^{64}$ & Spleen CD8- plasmacytoid DC \\
\hline
\end{tabular}

differences (Supplementary Fig. 5). These data suggest the increased urine $\lg A$ in aged mice is likely due to increased local production and not changes in the excretion rate of $\lg A$. To further determine if the increased amount of IgA was locally produced, we cultured bladders ex vivo for $24 \mathrm{~h}$, finding that aged bladders consistently produced higher concentrations of $\lg A$ than young bladders (Fig. 4g). Furthermore, we identified $\lg \mathrm{A}^{+} \mathrm{CD} 138^{+}$plasma cells associated with bTLT in aged bladders (Fig. 4b, d), indicating that these cells were locally differentiated and likely contributed to the high amounts of $\lg A$ in the urine from aged mice. Altogether, these findings indicate that bTLT in aged mice are functionally active in the local recruitment, activation, and differentiation of $B$ cells into IgA-secreting plasma cells.

Age-associated bTLT form independently of microbiota but require $\mathrm{TNFa}$

Both intrinsic and extrinsic factors influence age-associated changes in the immune system. Several lines of evidence point to the importance of microbial stimuli in TLT formation in other mucosal tissues including the lung and intestine. ${ }^{39,40}$ Furthermore, the intestinal microbiota significantly changes in old age and correlates with markers of inflamm-aging. ${ }^{41}$ To determine if microbes triggered age-associated bTLT formation, we examined bladders from aged germ free (GF) mice and age-matched, specific pathogen free (SPF) mice. Surprisingly, there were no differences in the number or size of bTLT in aged GF mice compared with SPF controls (Fig. 5a, b), demonstrating that a living microbiota is not required for age-associated TLT formation in the bladder. Tissue RNA-seq of young and aged bladders identified a number of cytokine and chemokine mediators that are likely involved in age-associated bTLT formation (Fig. 1c). In particular, we identified and validated increased expression of Tnf (TNFa, Fig. 5C), a pro-inflammatory cytokine that increases during aging and impacts age-related pathologies. ${ }^{23,24,39}$ While TNFa is required during acute immune responses, particularly to combat bacterial infection, excess or prolonged TNFa can lead to disease. The role of age-associated TNFa in many tissues, including the bladder, remain unknown. To test whether increased TNFa with age was a key driver of bTLT formation, we aged $\mathrm{TNFa}^{-1-}$ mice to 18-24 $\mathrm{m}$ and examined their bladders for bTLT formation. While aged $\mathrm{TNFa}^{-1-}$ mice had small, perivascular infiltrates in the bladder (Fig. 5b), they had fewer and smaller bTLT than agematched WT controls (Fig. 5c). Due to the rarity of bTLT in aged $\mathrm{TNFa}^{-1-}$ mice, we were unable to identify comparable structures by immunofluorescence. These data suggest that age-associated TNFa plays a significant role in the expansion and/or maturation of bTLT but may not be responsible for the initial recruitment of small numbers of lymphocytes to the aging bladder. In the context of our overall findings, age-associated TNFa is an important mediator reshaping the immune landscape in the aging bladder to promote the formation of bTLTs.

\section{DISCUSSION}

The consequences of immune aging on mucosal tissues such as the bladder are only beginning to be unraveled. ${ }^{21}$ To gain insight into the connection between aging, inflammation, and bladder disease, we sought to define how aging affects the bladder in an unperturbed state. At the tissue transcriptomic level, we found that nearly all highly significant changes point to an altered immune landscape being a fundamental feature of the aging bladder. The presence of lymphoid aggregates, composed of $B$ and $T$ cells organized into bTLT, underlie these tissue-level transcriptomic changes. Our findings suggest that age-associated inflammation, or inflamm-aging, could explain why aging is a risk factor for a plethora of bladder diseases. Bladder diseases are highly prevalent among women over 55 and continue to increase with age. ${ }^{9,21}$ For example, OAB affects $\sim 50 \%$ of women over age $65 .^{6}$ Inflamm-aging is proposed as a causative or exacerbating factor of $O A B$, and one study found 

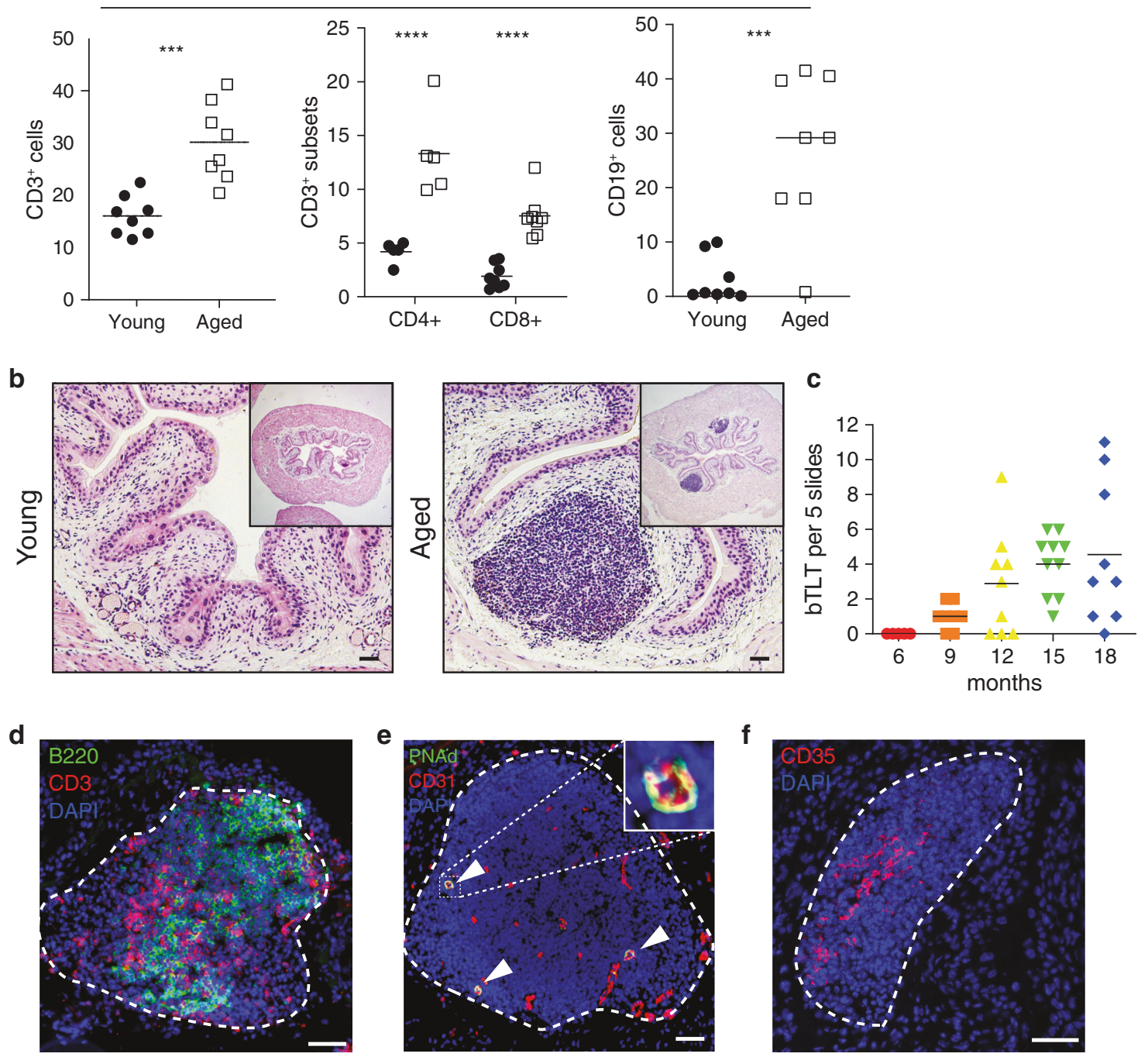

Fig. 3 Lymphoid infiltrates form bladder tertiary lymphoid tissues (bTLT) during aging. a Frequency of B cells, T cells, and T cell subsets among live $\mathrm{CD}_{4} 5^{+}$cells in young and aged bladders by flow cytometry. $n=5-8$ per group. $\mathbf{b}$ Representative H\&E images of young and aged bladders. c Number of bTLT over the life course of mice $n=5-10 /$ group. c Representative image of B cells (B220 ${ }^{+}$, green) and T cells (CD3 ${ }^{+}$, red) in segregated zones within bTLT in aged mice. d Representative image of $C D 31^{+}\left(\right.$red) PNAd $^{+}$(green) high endothelial venules (white arrowheads) within bTLT in aged mice. e Representative image of CD35 $5^{\text {hi }}$ follicular dendritic cell network within bTLT in aged mice. All nuclei stained with DAPI (blue). All scale bars, $50 \mu \mathrm{m}$.

that elevated urinary $C C L 2, C X C L 1$, and nerve growth factor correlated with age in $\mathrm{OAB}$ patients. ${ }^{12}$ Women over age 55 are also more susceptible to rUTIs. ${ }^{8}$ Mouse models of rUTIs have demonstrated that a history of chronic bacteriuria, characterized by severe inflammation, predisposes to a more severe secondary infection after antibiotic treatment. ${ }^{42}$ Interestingly, these mice develop lymphoid aggregates in the bladder, while those that resolve their infections do not. ${ }^{18}$ The composition and structure of these aggregates have not been described; thus, it is unknown whether they are similar to bTLT found in aged mice. Since aged mice harbor bTLT at steady state, the impact of these structures on the bladder tissue environment could be similar in these models. Our findings suggest that an altered or dysfunctional local immune repertoire in the aging bladder could explain why aging is a risk factor for multiple distinct bladder diseases. Considering the high prevalence of bladder diseases, an expanding aged population, and the relative lack of data on how established disease models differ with age, investigation of these bladder diseases in aged animals is warranted. Our work provides a framework establishing how the bladder differs between young and aged female mice at steady state, including the presence of bTLTs.

We also generated a single-cell resolution, transcriptomic map of resident immune cells within bladder tissue of both young and aged mice, identifying a plethora of immune cells and novel subsets. Prior single-cell studies have used the whole dissociated bladder, resulting in sparse immune cell analysis in this tissue. ${ }^{43-45}$ In aggregate, these studies have identified macrophages, NK cells, two sets of DCs, T cells, and monocytes. Using sCRNA-seq of enriched immune cell populations, we identified known bladder immune cell populations as well as novel sets of immune cells. In the myeloid lineage, we identified two distinct subsets of macrophages, four subsets of classical DCs, monocytes, plasmacytoid DCs, and migratory DCs from both young and aged mice in our study. Bladder macrophage subsets could be distinguished by expression of Retnla (also known as RELMa or Fizz1), a hallmark gene of an alternatively-activated macrophage (M2) phenotype. Macrophages typically play a role in tissue repair and homeostasis, but this function has not yet been investigated in the bladder. In aging, macrophages and other immune cells maintain tissue 


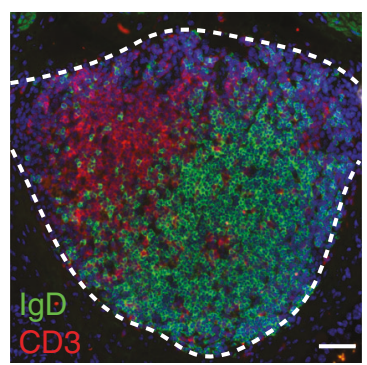

b

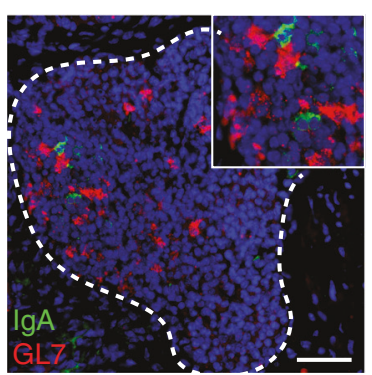

C

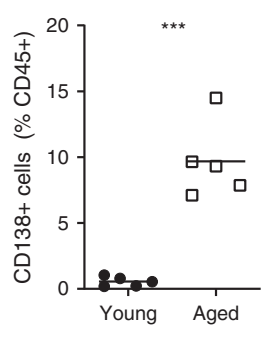

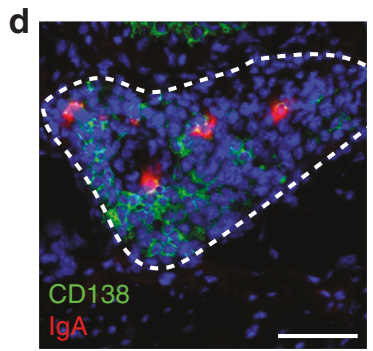
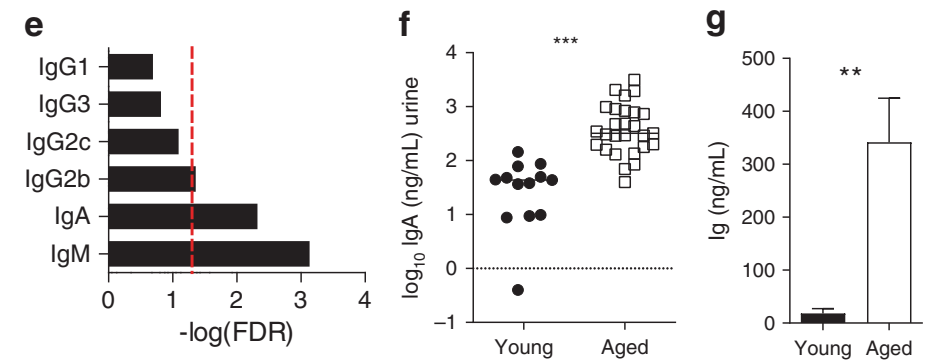

Fig. 4 bTLT are centers for B cell recruitment, activation, germinal center reactions, and plasma cell differentiation. a Representative image of naive $\mathrm{B}$ cells $\left(\mathrm{IgD}^{+}\right.$, green) and T cells $\left(\mathrm{CD}^{+}\right.$, red) within bTLT of aged bladders. $\mathbf{b}$ Representative image of IgA ${ }^{+}$cells within a $\mathrm{GL}^{+}$ (green) germinal center of a bTLT. c Frequency of live $\mathrm{CD} 45^{+} \mathrm{CD} 138^{+}$plasma cells in young and aged bladders by flow cytometry. $n=5 /$ group. d Representative image of $\lg \mathrm{A}^{+} \mathrm{CD} 138^{+}$plasma cells within bTLT of aged bladders. e FDR-adjusted $p$ values of IgM and class-switched isotypes from tissue RNA-seq of young and aged bladders. Red line, $p=0.05$. $\mathbf{f}$ Concentration of IgA in urine of young $(n=13)$ and aged $(n=27)$ mice. g Concentration of IgA in supernatants of young and bladders cultured ex vivo for $24 \mathrm{~h} . n=5 /$ group. All scale bars, $50 \mu \mathrm{m}$. ${ }^{* *} p<0.01$, ${ }^{* * *} p<$ 0.001. Mann-Whitney $U$ test.

homeostasis by clearing debris as cells die, become senescent, or accumulate damaged proteins. ${ }^{46}$ Impaired clearance of these cells by macrophages contributes to age-related pathologies and inflammation; in the aged bladder, failure to clear these cells could potentially lead to bTLT formation in an effort to compensate for this deficiency. We also identified a small group of macrophages expressing $\mathrm{CxCl} 13$ exclusively in the aged bladder. Since CXCL13 is a homeostatic lymphoid chemokine that attracts and organizes lymphocytes into SLOs and TLTs, these aged bladder macrophages likely play a key role in bTLT formation. While stromal cells are frequently a source of CXCL13 in other tissues, macrophages appear to take on this role in the aged bladder, demonstrating how the bladder may be a uniquely permissive tissue for TLT formation. In the lymphoid lineage, we identified $\mathrm{B}$ cells, NK cells, $\gamma \delta \mathrm{T}$ cells, $\mathrm{CD} 4^{+}$and $\mathrm{CD} 8^{+} \mathrm{T}$ cells, and ILC2. The most striking difference between young and aged bladders was the largely expanded $T$ and $B$ cell compartments that made up the bTLT described herein. In whole, these data demonstrate that the bladder immune system is likely much more complex than previously appreciated and dramatically altered during aging. This single-cell transcriptomic map of bladder immune cells will be a resource to further dissect the function of different immune cells in bladder health, disease, and aging.

bTLT in the aging bladder represents an unexpected and significant shift of the immune repertoire in the steady state bladder. Unlike other mucosae, the bladder does not normally harbor organized lymphoid tissue; the finding is otherwise only reported in diseased bladder tissue. Here we determined the cellular composition and structural organization of age-associated bTLT and demonstrate that they contain active GC reactions. A spectrum of $B$ cell states was identified by scRNA-seq in aged bladders, including naive, activated, and atypical/dysfunctional memory B cells (or ABCs), as well as a substantial increase in the number of antibody-producing plasma cells. B cell hematopoiesis is reduced in aging and results in oligoclonal expansion of existing B cells. ${ }^{47}$ In humans, these observations derive primarily from peripheral blood, and in mice, most studies focus on the spleen and bone marrow. Here we show that $B$ cells, including a large population of naive $B$ cells, accumulate within the bladder tissue. $B$ cell accumulation in tissues such as the bladder could be one factor contributing to the decline of B cells in the periphery. Our data showed that in aged mouse bladders, bTLT were capable of recruiting naive $B$ cells to undergo local activation, GC reactions, and plasma cell differentiation.

TLT are more likely to form in aged tissues in response to stimuli. For example, in the lung, aged mice more robustly form inducible bronchus-associated lymphoid tissue (iBALT) in response to cigarette smoke exposure. ${ }^{48}$ Aged mice also spontaneously develop distinct lymphocytic infiltrates in the lung, though these structures have not been formally defined to be iBALT. ${ }^{39}$ In contrast to our findings in the bladder, age-associated lung infiltrates are greatly reduced in aged GF mice, highlighting tissuespecific differences in potential triggers of TLT formation. In the intestine, isolated lymphoid follicles (ILFs) develop after birth and thus are considered TLTs. A greater number of both mature and immature ILFs are found in aged mice compared with young mice, and these ILFs contain altered cellular compositions. ${ }^{49}$ Interestingly, ILFs from aged mice produce lower amounts of chemokines yet produce more IgA than their younger counter parts. ILFs and their precursors are present in GF mice, ${ }^{50}$ but microbial colonization promotes the maturation of these $\mathrm{TLTs}^{40}{ }^{40}$ and intestinal microbiota are frequently the antigenic target of IgA produced therein. ${ }^{51}$ These studies establish that TLTs do not absolutely require a live microbiota, implying that they could target nonmicrobial antigens as well. GF mice are still exposed to environmental, dietary, and altered-self antigens that could drive the formation and maturation of these structures. In the urinary tract, microbial colonization also does not appear to be required to form age-associated bTLT, suggesting that in the urinary tract environment, these TLT typically target nonmicrobial antigens. One explanation may be an increased burden of altered proteins in the urinary environment, which is designed to store these toxic wastes. TLTs have also been found in the liver and kidneys of aged mice, and drivers of these TLTs also remain elusive. ${ }^{52,53}$ 


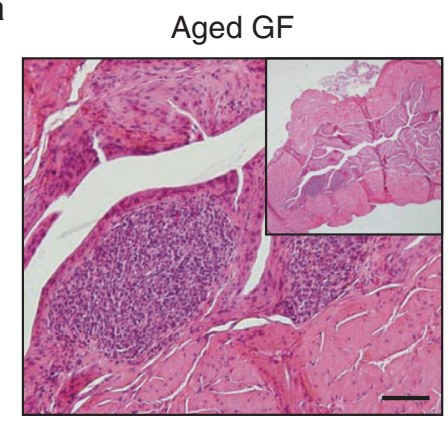

b

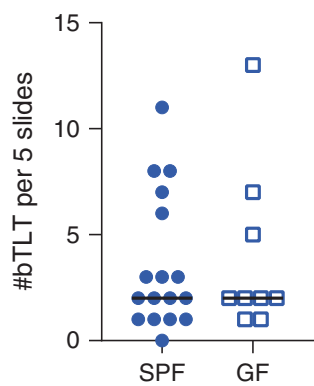

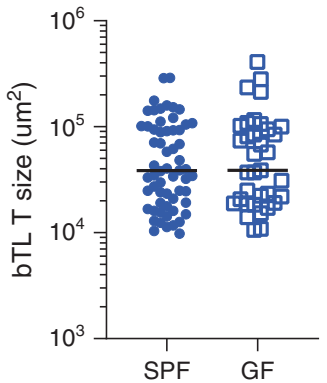

Aged TNF $\alpha^{-1-}$

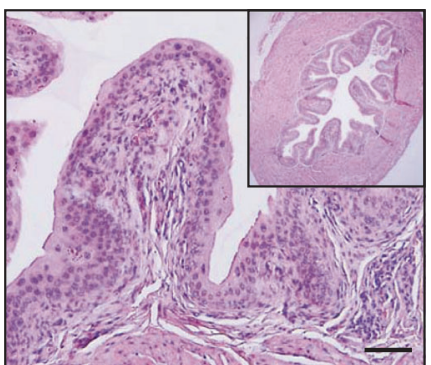

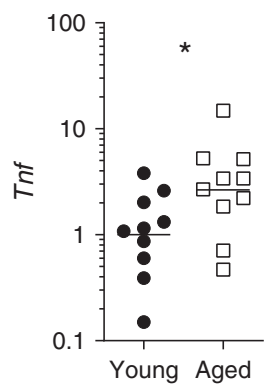

d

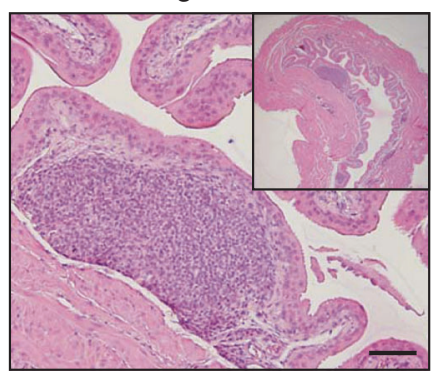

Aged WT

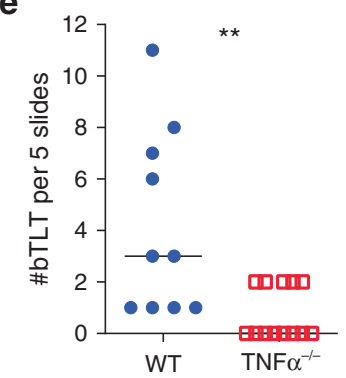

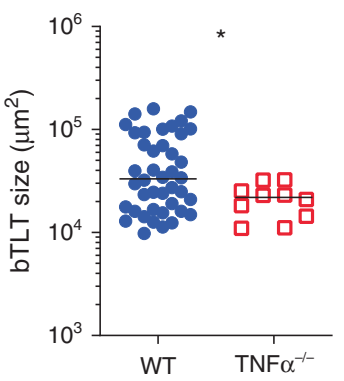

Fig. 5 bTLT size and number are independent of microbial status and dependent on age-associated TNFa. a Representative H\&E image of WT aged germ free (GF) bladders. b Number and size of bTLT found in WT specific pathogen free (SPF) and GF bladders. $n=9-17 / g r o u p$. c Relative expression of Tnf and in young and aged bladders by RT-qPCR. $n=10 /$ group. d Representative H\&E images of aged WT and TNF $\alpha^{-}-$ bladders. e Number and size of bTLT in aged WT and TNF $\alpha^{-/-}$bladders. Lines at median. ${ }^{*} p<0.05,{ }^{* *} p<0.01$, Mann-Whitney $U$-test. Scale bars, $100 \mu \mathrm{m}$.

Urine from aged mice had higher levels of $\lg A$, and aged bladders supported increased local IgA production. In humans, IgA is elevated in the serum during aging, ${ }^{54,55}$ and one study found IgA enriched in the urine proteome of elderly humans compared with young and middle-aged humans. ${ }^{56}$ While total IgA appears to be elevated in both mice and humans, generation of antigenspecific $\lg A$ is impaired. ${ }^{57}$ How $\lg A$ functions in the urinary tract remain ill-defined. IgA is increased during UTIs, particularly pyelonephritis, but whether it is protective in subsequent infections or dysregulated in those with rUTIs has not been shown. ${ }^{58}$ Patients with selective IgA deficiency are not reported to be more susceptible to UTIs, but this association has not been studied extensively and could be due to redundant or compensatory lgM. ${ }^{37}$ The target antigens of homeostatic or bTLT-derived IgA in the urinary tract is unknown, and could be a response to cumulative UTIs over the life, age-associated antigens, tissue damage from long-term urine exposure, the urinary microbiome, or age-associated dysfunctional B cell responses. Our data demonstrate that GF mice form similar numbers and sized bTLT, suggesting that nonmicrobial factors are likely causative. Further investigation of upstream factors influencing bTLT formation during aging and other urinary tract insults could begin to answer some of these questions.
We also identified a key, age-associated inflammatory cytokine, TNFa, as a mediator of bTLT formation in the aging bladder. Aged TNFa-deficient mice had reduced numbers and size of lymphoid aggregates compared with aged WT mice. TNFa is a mediator of inflamm-aging and has many roles in pathologic changes that arise during old age. ${ }^{23,24}$ Increased TNFa in centenarians is an independent risk factor for all-cause mortality, and chronic TNFa exposure impairs beneficial responses to pneumococcal pneumonia. ${ }^{23,39}$ However, TNFa is also essential for defense against pathogens, particularly extracellular bacteria. Indeed, TNFa is required for effective immune responses during UTIs. ${ }^{14,59}$ However, excess, inappropriate, or prolonged TNFa can promote pathologic changes leading to inflammatory disease and dysfunction during aging. TNFa contributes to IC/BPS, an inflammatory disorder of the bladder of unknown etiology that is more common in those over 50 years old. ${ }^{7,11,20,60}$ In a mouse model of IC/BPS, ectopic expression of TNFa in the bladder resulted in heighted pain sensitivity in response to bladder filling, ${ }^{61}$ but bTLT-like structures were not reported. These results could be due to factors such as age or sex and suggests that multiple processes are required to support these complex structures. Thus, a fine balance of protective inflammatory responses during acute insults and effective resolution of inflammation throughout life may be key to 
916

healthy aging. Considering our findings and the role of TNFa in inflamm-aging and bladder disease, clinical studies examining the relationship between age, TNFa levels, and bladder inflammation could provide further insight into these relationships.

While many questions about bTLT remain a mystery, this new finding demonstrates that aging leads to fundamental changes to the bladder characterized by a dramatically different immune landscape. Future work on the aging bladder should consider the presence of bTLT, as they underlie significant changes that likely impact multiple physiologic and homeostatic processes in the bladder. Finally, our single-cell transcriptomic map of bladder immune cells provides a new resource for studying the complexity of the bladder immune system.

\section{MATERIALS AND METHODS}

Mice

All experimental procedures were approved by the animal studies committee of Washington University in St. Louis School of Medicine (Animal Welfare Assurance \#A-3381-01) and McMaster University's Animal Research Ethics Board. 2- to 24-month-old C57BL6/J mice were obtained from the National Institute of Aging (NIA). NIA mice are bred and housed 5 mice/cage with wood shavings at Charles Rivers Laboratory on a $12 \mathrm{~h}$ light-dark cycle, at $67-73^{\circ} \mathrm{F}$ and $35-55 \%$ humidity, fed sterilized NIH31 food, and provided with water at pH7-7.5 with 4-6 ppm chlorine (see https://www.nia.nih.gov/research/dab/aged-rodent-colonieshandbook/barrier-environmental-information for more information). Upon receipt at Washington University, mice are housed at 5 mice/cage with autoclaved Bed-O'Cobs $1 / 4$ in. (Andersons Lab Bedding) on a $12 \mathrm{~h}$ light-dark cycle, at $68-74^{\circ} \mathrm{F}, 30-70 \%$ humidity, fed sterilized Purina Lab Diet 5053, and provided with sterilized tap water. At McMaster University, mice are housed at 5 mice/cage with Teklad 7090 sani-chips on a $12 \mathrm{~h}$ light-dark cycle, at $21-23^{\circ} \mathrm{C}$, $30-55 \%$ humidity, fed Teklad Irradiated Global 14\% protein maintenance diet, and provided with sterilized water and an exercise wheel. GF mice were housed at McMaster University Farncombe Family Axenic-Gnotobiotic Facility otherwise in the same conditions except fed Teklad S-2335 diet. Mice were maintained under specified pathogen-free conditions and monitored for mouse pathogens by on-going sentinel testing. Tnf ${ }^{-1-}$ (originally from Jackson Laboratories $\# 005540^{62}$ ), WT mice (originally from Jackson Laboratories \#000664), and GF mice were bred and aged to 18-24 months in house at McMaster University. ${ }^{39}$ To account for colony differences, $\mathrm{Tnf}^{-1-}$ mice and GF mice were compared with age-matched WT mice raised in the same facility. In all studies, mice from multiple cages, cohorts, and facilities (for data with WT only) were analyzed.

Histological and immunofluorescence analysis

Bladders were aseptically removed, cut in half, and fixed in $10 \%$ neutral buffered formalin or methacarn $(60 \%$ methanol, $30 \%$ chloroform, $10 \%$ acetic acid), embedded in paraffin, stained with hematoxylin and eosin (7211, Richard-Allen Scientific) and imaged on a Nanozoomer 2.0-HT system (Hamamatsu). Posterior bladder halves were compared within groups. Number and area of bTLTs were determined in five sections spaced $150 \mu \mathrm{m}$ apart using NDP. view2 software (Hamamatsu). Compact aggregates $>10,000 \mu \mathrm{m}^{2}$ were considered bTLTs. For immunofluorescence analysis, anterior bladders halves were embedded in OCT Compound (4583, TissueTek) and flash frozen. $7 \mu \mathrm{m}$ sections were fixed with 1:1 methanol-acetone, rehydrated in PBS, and blocked with Avidin/ Biotin Blocking Kit (SP2001, Vector Laboratories) followed by $1 \%$ BSA in PBS. Primary antibodies against B220 (13-0452, eBioscience), CD3 (14-0031-82, eBioscience) PNAd (120803, BioLegend), CD31 (ab28364, Abcam), CD138 (142511, BioLegend), CD35 (558768, BD Biosciences), GL7 (13-5902-81, eBioscience), IgA (1040-31, Southern Biotech), and IgD (1120-01, Southern Biotech) were incubated overnight at $4^{\circ}$ and detected with streptavidinconjugated and species-specific secondary antibodies followed by Hoechst dye. Slides were covere slipped with Prolong Gold antifade (P36930, Invitrogen) and imaged on Zeiss Axio Imager M2 microscope with Hamamatsu Flash4.0 camera using Zeiss Zen Pro software.

\section{Organ culture and IgA ELISA}

Bladders were aseptically removed, bisected, rinsed with PBS, and both halves cultured together in $500 \mu \mathrm{L} \mathrm{RPMI-1640} \mathrm{with} 10 \%$ FBS, $1 \%$ Pen/Strep (15-140-122, Gibco), $10 \mathrm{mM}$ HEPES, and $1 \%$ Glutamax (35030-061, Gibco). Supernatants were removed after $24 \mathrm{~h}$ and cleared of debris by centrifugation. IgA concentration in urines and culture media was determined by ELISA according to manufacturer protocol (88-50450-22, Invitrogen).

\section{Flow cytometry}

Bladders were aseptically removed, minced with scissors, and digested at $37^{\circ}$ for $30 \mathrm{~min}$ in RPMI- 1640 with $10 \mathrm{mM}$ HEPES, collagenase D (C5318, Sigma-Aldrich), and DNAse (10104159001, Sigma-Aldrich). Bladders were forced through a $70 \mu \mathrm{m}$ cell strainer (352350, Corning) and washed with 5\% FBS in PBS. Single cell suspensions were stained with anti-CD45-eFluor450 (48-0451-82, eBioscience), anti-CD3-APC (17-0032-82, eBioscience), anti-CD19PE (115511, BioLegend), anti-CD4-FITC (100405, BioLegend), antiCD8-PE/Cy7 (100721, BioLegend), anti-CD138-BrillantViolet605 (142515, BioLegend), and 7-AAD (420404, BioLegend). Data were acquired on LSR II flow cytometer (BD) and analyzed with FlowJo software v10.0. Gates were determined with isotype antibodies in bladder suspensions from young mice.

\section{Tissue RNA-sequencing}

RNA was purified from snap frozen, homogenized bladders with RNeasy Mini Kit (74101, Qiagen) and RNase-free DNase digestion kit (79254, Qiagen). Libraries were prepared with Ribo-Zero rRNA depletion kit (Illumina) and sequenced on a HiSeq3000 (Illumina). Reads were aligned to the Ensembl GRCm38.76 top-level assembly with STAR version 2.0.4b and gene counts were derived from the number of uniquely aligned unambiguous reads by Subread: featureCount version 1.4.5. Sequencing performance was then assessed for total number of aligned reads, total number of uniquely aligned reads, genes detected, ribosomal fraction, known junction saturation, and read distribution over known gene models with $\mathrm{RSeQC}$ version 2.3. All gene counts were then imported into the R/Bioconductor package EdgeR and TMM normalization size factors were calculated to adjust samples for differences in library size. Ribosomal features as well as any feature not expressed in at least three samples were excluded from further analysis, and TMM size factors were recalculated to create effective TMM size factors. The TMM size factors and the matrix of counts were then imported into the R/Bioconductor package Limma and weighted likelihoods based on the observed meanvariance relationship of every gene and sample were then calculated with the voomWithQualityWeights function. Generalized linear models were then created to test for gene level differential expression, and differentially expressed genes were then filtered for Benjamini-Hochberg FDR-adjusted $p$ values $\leq 0.05$ and absolute $\log _{2}$ fold-change $\geq 1$. The $\log _{2}$ fold-changes for all genes were then imported into the R/Bioconductor packages GAGE and Pathview to find pathways whose mean expression were perturbed versus background with Benajamini-Hochberg FDR-adjusted $p$ values $\leq 0.05$.

scRNA-seq and cell type identification

Bladders were digested with Multi-tissue dissociation kit (130-110201, Miltenyi) for $30 \mathrm{~min}$ at $37^{\circ}$. Bladder cells were filtered, washed, and incubated with magnetic CD45 microbeads (130-052-301, Miltenyi) and purified by two sequential positive selections 
(Supplementary Fig. 4a). Cells were washed and resuspended at appropriate concentrations for loading into 10X Chromium Controller using the Chromium Single Cell $5^{\prime}$ Library and Gel Bead Kit. Viability was determined by Trypan Blue with a hemocytometer. Reads were processed using cellranger v3.0.1. Filtered outputs were imported into the $\mathrm{R}$ package Seurat and combined. Default Seurat parameters were used unless otherwise noted. Strict filters of $>200$ genes/cell, $<5 \%$ mitochondrial reads/ cell, $<7500$ features/cell, and $<2500$ genes/cell were used. RunPCA was performed on the top 3000 variable genes from FindVariableFeatures. FindNeighbors and FindClusters were used with the first 40 principle components (PCs) and visualized using Uniform Manifold Approximation and Projection (Supplementary Fig. 2b). Three clusters drove variation attributed to PC1 (Supplementary Fig. 2c). Expression of urothelial specific gene Upk3a identified urothelial cells in two of these clusters; expression of Dcn identified stromal cells as the other cluster; and CD45 (Ptprc) expression identified the remaining cells as immune cells (Supplementary Fig. 2c). Urothelial and stromal cells were excluded from further analysis. PCA was again performed on the top 3000 variable genes of this subset. The first two PCs demonstrated balanced grouping of approximately three main groups of cell types (Supplementary Fig. 2c). Find Neighbors and FindClusters were used with the first 30 PCs of this subset, resulting in 21 cell clusters. FindAllMarkers was used with min.pct $=0.25$ to identify cluster-specific genes (Supplementary Table 2). Cell identities were determined by expression of canonical gene markers (Table 1 and Supplementary Fig. 3). The remaining cell types were identified by literature searches of top differentially expressed genes (Supplementary Table 2) and by loading all cluster markers into the Cluster Identity PRedictor (CIPR) ${ }^{25}$ (Supplementary Table 3) web tool (https://aekiz.shinyapps.io/ CIPR/). The dot product method was used with ImmGen as the reference database.

\section{RT-qPCR}

Bladders were flash frozen or stabilized in RNA Save (01-891-1A, Biological Industries) and RNA extracted using TRIzol reagent (15596018, Invitrogen) according to manufacturer protocol followed by gDNA digestion with TURBO DNA-free kit (AM1907, Invitrogen). cDNA was generated using Superscript II Reverse Transcriptase (18064014, Invitrogen). qPCR was performed with SsoAdvanced Universal SYBR Green Supermix (1725275, Bio-Rad) on a CFX96 Touch Real-Time PCR Detection System (Bio-Rad). Fold-changes were calculated using $\Delta \Delta C t$ method and normalized internally to $18 \mathrm{~S}$ expression.

\section{Statistical analyses}

Statistical tests were performed in GraphPad Prism 8. Datasets were evaluated for normality and lognormality with AndersonDarling, D'Agostino-Pearson, Shipiro-Wilk, and KolmogorovSmirno tests. Lognormal distributions were log-transformed and analyzed as a parametric distribution. Unpaired $t$-tests (with Welch's correction where appropriate) or two-way ANOVA with Bonferroni post-tests were used for parametric data, and Mann-Whitney $U$ test or Kruskal-Wallis with Dunn's multiple comparison tests were used for non-parametric data. $p<0.05$ was considered significant. Data points represent individual animals. Lines represent the mean for normal distributions, geometric mean for log-normal distributions, or median for nonparametric distributions. Error bars represent SEM. For RNA-seq experiments, Benjamini-Hochberg FDR-adjusted $p$ values $\leq 0.05$ were considered significant.

\section{DATA AVAILABILITY}

All sequencing data is available at GSE149571.

\section{ACKNOWLEDGEMENTS}

We thank Drs. Deborah Frank, Jason Mills, and Paula Saz-Leal for editorial comments, the Genome Technology Access Center (GTAC) for performing and processing sequencing data, and Eric Tycksen for bioinformatic analysis and statistical explanations of tissue RNA-seq data. This work was funded in part by $\mathrm{NIH}$ grants R01 AG052494, P20 DK119840, and R56 AG064634 to I.U.M.; T32 GM007200 and T32 Al007172 to M.M.L.; CIHR \#153414 to D.M.E.B.; Deutsche Forschungsgemeinschaft fellowship \#SCHU3131/1-1 to C.S.; Ontario Early Researchers award to E.N.D.; NIH Shared Instrumentation Grant S10 RR0275523 to Alafi Neuroimmaging Core; and P30 CA91842 and UL1 TR002345 to G.T.A.C.

\section{AUTHOR CONTRIBUTIONS}

Conceptualization M.M.L., C.W., I.U.M. Methodology M.M.L., C.W., I.U.M., D.M.E.B. Investigation M.M.L., C.W., C.S., E.N.D. Data analysis M.M.L. Resources I.U.M., D.M.E.B. Supervision C.W., D.M.E.B., I.U.M. Funding I.U.M., D.M.E.B. Visualization M.M.L. Writingoriginal draft M.M.L., I.U.M. Writing-review and editing M.M.L., C.W., D.M.E.B., I.U.M.

\section{ADDITIONAL INFORMATION}

The online version of this article (https://doi.org/10.1038/s41385-020-0290-x) contains supplementary material, which is available to authorized users.

Competing interests: The authors declare no competing interests.

Publisher's note Springer Nature remains neutral with regard to jurisdictional claims in published maps and institutional affiliations.

\section{REFERENCES}

1. Franceschi, C. \& Campisi, J. Chronic inflammation (inflammaging) and its potential contribution to age-associated diseases. J. Gerontol. A Biol. Sci. Med. Sci. 69(Suppl 1), S4-9 (2014).

2. Niccoli, T. \& Partridge, L. Ageing as a risk factor for disease. Curr. Biol. 22, R741-R752 (2012).

3. McHugh, D. \& Gil, J. Senescence and aging: causes, consequences, and therapeutic avenues. J. Cell Biol. 217, 65-77 (2018).

4. Abraham, S. N. \& Miao, Y. The nature of immune responses to urinary tract infections. Nat. Rev. Immunol. 15, 655-663 (2015).

5. Wu, X. R., Kong, X. P., Pellicer, A., Kreibich, G. \& Sun, T. T. Uroplakins in urothelial biology, function, and disease. Kidney Int. 75, 1153-1165 (2009).

6. Nygaard, I. et al. Prevalence of symptomatic pelvic floor disorders in US women. JAMA 300, 1311-1316 (2008).

7. Koziol, J. A., Adams, H. P. \& Frutos, A. Discrimination between the ulcerous and the nonulcerous forms of interstitial cystitis by noninvasive findings. J. Urol. 155, 87-90 (1996).

8. Suskind, A. M. et al. Incidence and management of uncomplicated recurrent urinary tract infections in a national sample of women in the United States. Urology 90, 50-55 (2016).

9. Maserejian, N. N. et al. Incidence of lower urinary tract symptoms in a populationbased study of men and women. Urology 82, 560-564 (2013).

10. $\mathrm{Ma}, \mathrm{E}$. et al. A multiplexed analysis approach identifies new association of inflammatory proteins in patients with overactive bladder. Am. J. Physiol. Ren. Physiol. 311, F28-F34 (2016).

11. Grundy, L., Caldwell, A. \& Brierley, S. M. Mechanisms underlying overactive bladder and interstitial cystitis/painful bladder syndrome. Front Neurosci. 12, 931 (2018).

12. Tyagi, P. et al. Association of inflammaging (inflammation + aging) with higher prevalence of OAB in elderly population. Int. Urol. Nephrol. 46, 871-877 (2014).

13. Mora-Bau, G. et al. Macrophages subvert adaptive immunity to urinary tract infection. PLoS Pathog. 11, e1005044 (2015).

14. Schiwon, M. et al. Crosstalk between sentinel and helper macrophages permits neutrophil migration into infected uroepithelium. Cell 156, 456-468 (2014).

15. Pitzalis, C., Jones, G. W., Bombardieri, M. \& Jones, S. A. Ectopic lymphoid-like structures in infection, cancer and autoimmunity. Nat. Rev. Immunol. 14, 447-462 (2014).

16. Aloisi, F. \& Pujol-Borrell, R. Lymphoid neogenesis in chronic inflammatory diseases. Nat. Rev. Immunol. 6, 205-217 (2006).

17. Chen, S. C. et al. Ectopic expression of the murine chemokines CCL21a and $\mathrm{CCL} 21 \mathrm{~b}$ induces the formation of lymph node-like structures in pancreas, but not skin, of transgenic mice. J. Immunol. 168, 1001-1008 (2002).

18. Hannan, T. J., Mysorekar, I. U., Hung, C. S., Isaacson-Schmid, M. L. \& Hultgren, S. J. Early severe inflammatory responses to uropathogenic E. coli predispose to chronic and recurrent urinary tract infection. PLoS Pathog. 6, e1001042 (2010). 
19. Koti, M. et al. Tertiary lymphoid structures associate with tumour stage in urothelial bladder cancer. Bladder Cancer 3, 259-267 (2017).

20. Maeda, D. et al. Hunner-type (classic) interstitial cystitis: a distinct inflammatory disorder characterized by pancystitis, with frequent expansion ofcClonal B-cells and epithelial denudation. PloS ONE 10, e0143316 (2015).

21. Ellsworth, P., Marschall-Kehrel, D., King, S. \& Lukacz, E. Bladder health across the life course. Int J. Clin. Pract. 67, 397-406 (2013).

22. Ingersoll, M. A. \& Albert, M. L. From infection to immunotherapy: host immune responses to bacteria at the bladder mucosa. Mucosal. Immunol. 6, 1041-1053 (2013).

23. Bruunsgaard, H., Andersen-Ranberg, K., Hjelmborg, J., Pedersen, B. K. \& Jeune, B. Elevated levels of tumor necrosis factor alpha and mortality in centenarians. $\mathrm{Am}$. J. Med. 115, 278-283 (2003).

24. Giovannini, S. et al. Interleukin-6, C-reactive protein, and tumor necrosis factoralpha as predictors of mortality in frail, community-living elderly individuals. J. Am. Geriatr. Soc. 59, 1679-1685 (2011).

25. Ekiz, H. A. et al. MicroRNA-155 coordinates the immunological landscape within murine melanoma and correlates with immunity in human cancers. JCI Insight 4, e126543 (2019)

26. Heng, T. S. \& Painter, M. W., Immunological Genome Project C. The Immunological Genome Project: networks of gene expression in immune cells. Nat. Immunol. 9, 1091-1094 (2008).

27. Sivick, K. E., Schaller, M. A., Smith, S. N. \& Mobley, H. L. The innate immune response to uropathogenic Escherichia coli involves IL-17A in a murine model of urinary tract infection. J. Immunol. 184, 2065-2075 (2010).

28. Liu, W., Evanoff, D. P., Chen, X. \& Luo, Y. Urinary bladder epithelium antigen induces CD8 + T cell tolerance, activation, and autoimmune response. J. Immunol. 178, 539-546 (2007).

29. Rousseau, M. et al. Bladder catheterization increases susceptibility to infection that can be prevented by prophylactic antibiotic treatment. JCI Insight 1, e88178 (2016).

30. Chan, C. Y., St John, A. L. \& Abraham, S. N. Mast cell interleukin-10 drives localized tolerance in chronic bladder infection. Immunity 38, 349-359 (2013).

31. Trivedi, N. et al. Liver is a generative site for the $b$ cell response to Ehrlichia muris. Immunity 51, 1088-1101.e5 (2019).

32. Sullivan, R. T. et al. FCRL5 delineates functionally impaired memory b cells associated with plasmodium falciparum exposure. PLoS Pathog. 11, e1004894 (2015).

33. Klein, R. D. et al. Transitional cell hyperplasia and carcinomas in urinary bladders of transgenic mice with keratin 5 promoter-driven cyclooxygenase-2 overexpression. Cancer Res. 65, 1808-1813 (2005).

34. Jones, G. W. \& Jones, S. A. Ectopic lymphoid follicles: inducible centres for generating antigen-specific immune responses within tissues. Immunology 147, 141-151 (2016).

35. Mesin, L., Ersching, J. \& Victora, G. D. Germinal center B cell dynamics. Immunity 45, 471-482 (2016).

36. Uhlen, M. et al. Proteomics. Tissue-based map of the human proteome. Science 347, 1260419 (2015)

37. Floege, J., Boddeker, M., Stolte, H. \& Koch, K. M. Urinary lgA, secretory IgA and secretory component in women with recurrent urinary tract infections. Nephron 56, 50-55 (1990).

38. Yanagihara, T. et al. Age-dependent decrease of polymeric lg receptor expression and IgA elevation in ddY mice: a possible cause of IgA nephropathy. Lab. Investig. 84, 63-70 (2004).

39. Thevaranjan, N. et al. Age-associated microbial dysbiosis promotes intestinal permeability, systemic inflammation, and macrophage dysfunction. Cell Host Microbe 21, 455-466. e454 (2017).

40. Pabst, O. et al. Adaptation of solitary intestinal lymphoid tissue in response to microbiota and chemokine receptor CCR7 signaling. J. Immunol. 177, 6824-6832 (2006).

41. Claesson, M. J. et al. Gut microbiota composition correlates with diet and health in the elderly. Nature 488, 178-184 (2012).
42. O'Brien, V. P. et al. A mucosal imprint left by prior Escherichia coli bladder infection sensitizes to recurrent disease. Nat. Microbiol. 2, 16196 (2016).

43. $\mathrm{Yu}, \mathrm{Z}$. et al. Single-cell transcriptomic map of the human and mouse bladders. J. Am. Soc. Nephrol. 30, 2159-2176 (2019).

44. Han, X. et al. Mapping the mouse cell atlas by microwell-Seq. Cell 173, 1307 (2018).

45. Tabula Muris, C., Overall c, Logistical c, Organ c, processing, Library p. et al. Singlecell transcriptomics of 20 mouse organs creates a Tabula Muris. Nature 562, 367-372 (2018)

46. Ovadya, Y. et al. Impaired immune surveillance accelerates accumulation of senescent cells and aging. Nat. Commun. 9, 5435 (2018).

47. Riley, R. L., Khomtchouk, K. \& Blomberg, B. B. Age-associated B cells (ABC) inhibit B lymphopoiesis and alter antibody repertoires in old age. Cell Immunol. 321, 61-67 (2017).

48. John-Schuster, G. et al. Inflammaging increases susceptibility to cigarette smokeinduced COPD. Oncotarget 7, 30068-30083 (2016).

49. McDonald, K. G., Leach, M. R., Huang, C., Wang, C. \& Newberry, R. D. Aging impacts isolated lymphoid follicle development and function. Immun. Ageing 8, 1 (2011).

50. Hamada, $H$. et al. Identification of multiple isolated lymphoid follicles on the antimesenteric wall of the mouse small intestine. J. Immunol. 168, 57-64 (2002).

51. Sterlin, D., Fadlallah, J., Slack, E. \& Gorochov, G. The antibody/microbiota interface in health and disease. Mucosal Immunol. 13, 3-11 (2020).

52. Sato, Y. et al. Heterogeneous fibroblasts underlie age-dependent tertiary lymphoid tissues in the kidney. $\mathrm{JCl}$ Insight 1, e87680 (2016)

53. Singh, P. et al. Lymphoid neogenesis and immune infiltration in aged liver. Hepatology 47, 1680-1690 (2008).

54. Radl, J., Sepers, J. M., Skvaril, F., Morell, A. \& Hijmans, W. Immunoglobulin patterns in humans over 95 years of age. Clin. Exp. Immunol. 22, 84-90 (1975).

55. Tietz, N. W., Shuey, D. F. \& Wekstein, D. R. Laboratory values in fit aging individuals-sexagenarians through centenarians. Clin. Chem. 38, 1167-1185 (1992).

56. Bakun, M. et al. Urine proteomes of healthy aging humans reveal extracellular matrix (ECM) alterations and immune system dysfunction. Age 36, 299-311 (2014).

57. Thoreux, K., Owen, R. L. \& Schmucker, D. L. Intestinal lymphocyte number, migration and antibody secretion in young and old rats. Immunology 101, 161-167 (2000)

58. James-Ellison, M. Y., Roberts, R., Verrier-Jones, K., Williams, J. D. \& Topley, N. Mucosal immunity in the urinary tract: changes in slgA, FSC and total IgA with age and in urinary tract infection. Clin. Nephrol. 48, 69-78 (1997).

59. $\mathrm{Yu}, \mathrm{L}$. et al. Mucosal infection rewires TNFa signaling dynamics to skew susceptibility to recurrence. Elife 8, e46677 (2019).

60. Simon, L. J., Landis, J. R., Erickson, D. R. \& Nyberg, L. M. The Interstitial Cystitis Data Base Study: concepts and preliminary baseline descriptive statistics. Urology 49 (5A Suppl), 64-75 (1997).

61. Yang, W., Searl, T. J., Yaggie, R., Schaeffer, A. J. \& Klumpp, D. J. A. MAPP Network study: overexpression of tumor necrosis factor-alpha in mouse urothelium mimics interstitial cystitis. Am. J. Physiol. Ren. Physiol. 315, F36-F44 (2018).

62. Pasparakis, M., Alexopoulou, L., Episkopou, V. \& Kollias, G. Immune and inflammatory responses in TNF alpha-deficient mice: a critical requirement for TNF alpha in the formation of primary B cell follicles, follicular dendritic cell networks and germinal centers, and in the maturation of the humoral immune response. J. Exp. Med 184, 1397-1411 (1996).

63. Bando, J. K., Nussbaum, J. C., Liang, H. E. \& Locksley, R. M. Type 2 innate lymphoid cells constitutively express arginase-I in the naive and inflamed lung. J. Leukoc. Biol. 94, 877-884 (2013).

64. Miller, J. C. et al. Deciphering the transcriptional network of the dendritic cell lineage. Nat. Immunol. 13, 888-899 (2012). 